\title{
A New Way to Calculate Gravity Force from Mass Total Energy
}

\author{
Keli Kou \\ Beijing, China \\ Email: kelikou@163.com
}

How to cite this paper: Kou, K.L. (2021) A New Way to Calculate Gravity Force from Mass Total Energy. Open Access Library Journal, 8: e7987.

https://doi.org/10.4236/oalib.1107987

Received: September 21, 2021

Accepted: October 18, 2021

Published: October 21, 2021

Copyright $\odot 2021$ by author(s) and Open Access Library Inc.

This work is licensed under the Creative Commons Attribution International License (CC BY 4.0).

http://creativecommons.org/licenses/by/4.0/

\section{(c) (i) Open Access}

\begin{abstract}
This paper introduces a new way to calculate gravity force from mass total energy, instead of from matter of mass. By introducing total energy $\left(E_{1}\right.$ and $\left.E_{2}\right)$ of the two objects into Newton's gravitational theory, Newton's gravitational formula can be modified from $F=G M_{1} M_{2} / r^{2}$ as $F=G E_{1} E_{2} /\left(C^{4} r^{2}\right) . E_{1}$ and $E_{2}$ are the total energy of the two objects and $C$ is the light speed. Then this formula can be further modified as $F=G M_{1} M_{2}\left(1+V^{2} / C^{2}+V^{A} /\left(4 C^{4}\right)\right) / r^{2}$. $V$ is the relative speed between the two objects. In order to test this formula, the paper uses it to calculate the Perihelion Precession of Mercury and light deflection near the Sun. The calculation result shows that Modified Newton's Gravity theory and General Relative theory have the same accuracy to calculate planets orbit and light deflection. It also shows that the gravitational force between two objectives with high relative speed is a bit bigger than that with low relative speed. When this speed is up to light speed, the gravity force is 2.25 times bigger than that with zero speed.
\end{abstract}

\section{Subject Areas}

Classical Mechanics, Classical Physics, Mechanics, Modern Physics,

Theoretical Physics

\section{Keywords}

MOND Theories of Gravity, Mercury Precession, Light Deflection

\section{Introduction}

From Newton's gravitational force, the planets' orbit can be calculated as an oval orbit [1]. But the Perihelion Precession of Mercury and light deflection near the Sun are the two examples that can't be explained from Newton's theory. The main reason is that Newton's theory did not consider the total energy of the two 
objects in the formula of Newton's gravitational force, but only consider total mass of the two objects. This article tries to modify Newton's gravitational formula by introducing an object's total energy and relative speed into Newton's gravitational formula. The modified Newton's gravitational formula will be tested from the two experiments' observations, including the Perihelion Precession of Mercury and light deflection near the Sun. The following calculation approved that the modified Newton's gravitational formula can well explain the Perihelion Precession of Mercury and light deflection near the Sun, with the same result from the calculation of general relativity theory.

\section{The Modified Newton's Gravity Force Formula, Considering Total Energy of the Two Objects}

According to Newton's gravity formula, the gravity force of the two objects is:

$$
F=\frac{G M_{1} M_{2}}{r^{2}} .
$$

This formula shows that gravity force comes from the mass of objects. According to Einstein's Mass Energy Equation, the mass internal energy is $E=$ $M C^{2}$. The mass dynamic energy is $E_{k}=M V^{2} / 2$. It is estimated that gravity force may be related to the total energy of objects, not just the mass of objects. In this paper, it is recommended to use the object's total energy to calculate the gravity force, which will give more accurate results to calculate Mercury Precession and Light Deflection. The modified formula is as below:

$$
F=\frac{G E_{1} E_{2}}{C^{4} r^{2}}
$$

In this formula, $E_{1}$ and $E_{2}$ are the total energy of the two objects. $C$ is light speed. According to Einstein's Mass-Energy Equation, the total energy of the mass includes internal energy and dynamic energy. The formula of total energy for the two objects is shown as below:

$$
\begin{aligned}
& E_{1}=M_{1} C^{2}+\frac{M_{1} V^{2}}{2} ; \\
& E_{2}=M_{2} C^{2}+\frac{M_{2} V^{2}}{2} .
\end{aligned}
$$

Then Formula (2.1) can be modified as:

$$
F=\frac{G\left(M_{1} C^{2}+\frac{M_{1} V^{2}}{2}\right)\left(M_{2} C^{2}+\frac{M_{2} V^{2}}{2}\right)}{C^{4} r^{2}} .
$$

To simplify the above formula, we have:

$$
F=\frac{G M_{1} M_{2}\left(1+\frac{V^{2}}{C^{2}}+\frac{V^{4}}{4 C^{4}}\right)}{r^{2}}
$$

For those objects with low speed, $V^{4} / C^{4} \approx 0$, then the $V^{4} / C^{4}$ can be ignored, 
and Formula (2.2) can be simplified as below:

$$
F \approx \frac{G M_{1} M_{2}\left(1+\frac{V^{2}}{C^{2}}\right)}{r^{2}}
$$

Formulas (2.2) and (2.3) are the modified Gravity formula, conducted from Formula (2.1). The next parts of the paper will provide 3 evidences to approve this formula.

\section{Test the Modified Newton's Theory from the Perihelion Precession of Mercury}

\subsection{To Calculate the Planet's Orbit from Newton's Gravitational Theory}

From Newton's dynamic theory, planets orbit can be calculated from the below formula [1] [2]:

$$
\begin{gathered}
\frac{\mathrm{d} \varphi}{\mathrm{d} t}=\frac{L}{r^{2}} \\
\frac{1}{2}\left(\frac{\mathrm{d} r}{\mathrm{~d} t}\right)^{2}=E+\frac{G M}{r}-\frac{L^{2}}{2 r^{2}}
\end{gathered}
$$

Formula (3.1) shows the planet's angular momentum conservation, and Formula (3.2) shows the planet's energy conservation. $L$ is the angular momentum and $E$ is the energy. $r$ is the distance from planet to the center of polar coordinates. The $\varphi$ is the angle of the planet in polar coordinates. To cancel $t$, then we have the planet orbit formula:

$$
\frac{1}{2}\left[\frac{\mathrm{d}}{\mathrm{d} \varphi}\left(\frac{1}{r}\right)\right]^{2}=\frac{E}{L^{2}}-\frac{1}{2 r^{2}}+\frac{G M}{r L^{2}}
$$

To derivative the above formula at both sides, then we have:

$$
\frac{\mathrm{d}^{2}}{\mathrm{~d} \varphi^{2}}\left(\frac{1}{r}\right)+\frac{1}{r}=\frac{G M}{L^{2}}
$$

To replace the variable as below:

$$
u=\frac{G M}{r} .
$$

Formula (3.3) can be modified as:

$$
\frac{\mathrm{d}^{2} u}{\mathrm{~d} \varphi^{2}}+u=\left(\frac{G M}{L}\right)^{2}
$$

This equation is from Newton's theory to describe the planet's orbit [1] [2]. The solution of the equation is:

$$
\begin{gathered}
u=\left(\frac{G M}{L}\right)^{2}(1+e \cos \varphi) \\
r=\frac{L^{2}}{G M} * \frac{1}{1+e \cos \varphi}
\end{gathered}
$$


The above formula shows the planet's orbit is a closed oval, according to Newton's theory.

But from the experiments' observation, the planet orbit is not a closed oval. At the perihelion, there is precession, especially for Mercury. Every 100 years, there is 43" precession for Mercury, which can't be explained by Newton's theory.

This article will use the modified Newton's theory to explain this precession of $43^{\prime \prime}$.

\subsection{To Calculate the Perihelion Precession of Mercury from the Modified Newton's Gravitational Formula}

For the object with a speed much slower than light speed, the modified Newton's gravitational formula should be from Formula (2.3), and is shown as below [3] [4]:

$$
F=\frac{G M m}{r^{2}}\left(1+\frac{V^{2}}{C^{2}}\right)
$$

$F$ is the gravitational force between sun and planet; $M$ is the mass of the sun; $m$ is the mass of planet; $r$ is the distance between sun and planet; $V$ is the planet speed relative to the sun.

Based on Newton's dynamic theory and modified Newton's gravitational formula, the plant orbit comes from the two equations as below.

$$
\begin{gathered}
\frac{\mathrm{d} \varphi}{\mathrm{d} t}=\frac{L}{r^{2}} \\
\frac{1}{2}\left(\frac{\mathrm{d} r}{\mathrm{~d} t}\right)^{2}=E+\frac{G M}{r^{2}}\left(1+\frac{V^{2}}{C^{2}}\right)-\frac{L^{2}}{2 r^{2}}
\end{gathered}
$$

$L$ is the angular momentum; $E$ is the energy; $V$ is the mercury speed to the sun.

According to dynamic theory, the planet speed can be resolved as the speed of tangential direction and radial direction.

$$
V^{2}=V_{\text {tangential }}^{2}+V_{\text {radial }}^{2}=\left(\frac{\mathrm{d} r}{\mathrm{~d} t}\right)^{2}+\left(r \frac{\mathrm{d} \varphi}{\mathrm{d} t}\right)^{2}=\left(\frac{\mathrm{d} r}{\mathrm{~d} t}\right)^{2}+\left(\frac{L}{r}\right)^{2}
$$

From above equation, Formula (3.10) can be written as:

$$
\begin{gathered}
\frac{1}{2}\left(\frac{\mathrm{d} r}{\mathrm{~d} t}\right)^{2}=E+\frac{G M}{r}+\frac{G M}{r C^{2}}\left[\left(\frac{\mathrm{d} r}{\mathrm{~d} t}\right)^{2}+\left(\frac{L}{r}\right)^{2}\right]-\frac{L^{2}}{2 r^{2}} \\
\left(\frac{1}{2}-\frac{G M}{r C^{2}}\right)\left(\frac{\mathrm{d} r}{\mathrm{~d} t}\right)^{2}=E+\frac{G M}{r}+\frac{G M}{r C^{2}} \frac{L^{2}}{r^{2}}-\frac{L^{2}}{2 r^{2}}
\end{gathered}
$$

In Formula (3.11),

$\frac{1}{2} \gg \frac{G M}{r C^{2}}$, Hence the part $\frac{G M}{r C^{2}}$ can be ignored and be deleted.

Then Formula (3.11) can be simplified and be written as: 


$$
\frac{1}{2}\left(\frac{\mathrm{d} r}{\mathrm{~d} t}\right)^{2}=E+\frac{G M}{r}+\frac{G M}{r} \frac{L^{2}}{(r C)^{2}}-\frac{L^{2}}{2 r^{2}}
$$

To combine Formulas (3.9) and (3.12), and delete $t$ in formula, then we have planet orbit equation:

$$
\frac{1}{2} \frac{L^{2}(\mathrm{~d} r)^{2}}{r^{4}(\mathrm{~d} \varphi)}=E-\frac{L^{2}}{2 r^{2}}+\frac{G M}{r}+\frac{G M}{r^{3}} \frac{L^{2}}{C^{2}}
$$

To derivative the Equation (3.13) at both sides, then we have:

$$
\frac{L^{2}}{2} \frac{\mathrm{d}^{2}}{\mathrm{~d} \varphi^{2}}\left(\frac{1}{r}\right)+\frac{L^{2}}{r}=G M+\frac{3 G M L^{2}}{r^{2} C^{2}}
$$

To make a replacement as below:

$$
u=\frac{G M}{r}
$$

The above equation can be written as:

$$
\frac{\mathrm{d}^{2} u}{\mathrm{~d} \varphi^{2}}+u=\left(\frac{G M}{L}\right)^{2}+\frac{3 u^{2}}{c^{2}}
$$

This equation is the same as the equation from general relative theory [5] [6]. According to Newton's theory, the planet orbit should be as below equation [5] [6]:

$$
\frac{\mathrm{d}^{2} u}{\mathrm{~d} \varphi^{2}}+u=\left(\frac{G M}{L}\right)^{2}
$$

The resolution of Equation (3.5) is:

$$
\begin{gathered}
u=\left(\frac{G M}{L}\right)^{2}(1+e \cos \theta) \\
r=\frac{L^{2}}{G M} * \frac{1}{1+e \cos \theta}
\end{gathered}
$$

Obviously, it is a closed oval orbit. And $e$ is the eccentricity of the orbit.

According to modified Newton's gravitational Formula (3.14), it shows that Mercury orbit is not a closed oval. Compared with Equation (3.5), it has an additional part $3 u^{2} / C^{2}$. This additional part is from the modification of Newton's Theory. For sun, there is $G M=1.5 \times 10^{3} \mathrm{~m}$. The radial of the mercury is $r=5 \times$ $10^{10} \mathrm{~m}$. The order of the $\mathrm{u}$ is:

$$
u=\frac{G M}{r} \sim 10^{-7}
$$

From Equation (3.6), we can find $\left(\frac{G M}{L}\right)^{2}$ and u has the same order:

$$
\left(\frac{G M}{L}\right)^{2} \sim 10^{-7} \text {. }
$$

From the above discussion, the additional part of the equation has a much lower order: 


$$
\frac{3 u^{2}}{C^{2}} \sim 10^{-30} .
$$

The additional part is so small, that we can treat resolution of Equation (3.6) as the zero order resolution of Equation (3.14).

To change the non linear Equation (3.14) to linear equation, we take zero order solution into Equation (3.14). Then we have the equation as below:

$$
\frac{\mathrm{d}^{2} u}{\mathrm{~d} \varphi^{2}}+u=\left(\frac{G M}{L}\right)^{2}+\frac{3}{c^{2}}\left[\left(\frac{G M}{L}\right)^{2}(1+e \cos \varphi)\right]^{2} .
$$

The above equation can be written as:

$$
\begin{aligned}
\frac{\mathrm{d}^{2} u}{\mathrm{~d} \varphi^{2}}+u= & \left(\frac{G M}{L}\right)^{2}+\frac{3}{c^{2}}\left(\frac{G M}{L}\right)^{4}+\frac{6}{c^{2}}\left(\frac{G M}{L}\right)^{4} e \cos \varphi \\
& +\frac{3}{C^{2}}\left(\frac{G M}{L}\right)^{4}(e \cos \varphi)^{2}
\end{aligned}
$$

As the eccentricity of Mercury orbit is very small, $e^{2} \ll 1$.

To compare the two constants, we have: $\left(\frac{G M}{L}\right)^{2} \gg \frac{3}{C^{2}}\left(\frac{G M}{L}\right)^{4}$.

To ignore the small constant,

$$
\frac{3}{C^{2}}\left(\frac{G M}{L}\right)^{4} \text { and } \frac{3}{C^{2}}\left(\frac{G M}{L}\right)^{4}(e \cos \varphi)^{2} .
$$

Equation (3.15) can be written as:

$$
\frac{\mathrm{d}^{2} u}{\mathrm{~d} \varphi^{2}}+u \approx\left(\frac{G M}{L}\right)^{2}+\frac{6}{C^{2}}\left(\frac{G M}{L}\right)^{4} e \cos \varphi
$$

We separate the solution of the liner equation into two parts: $u=u_{1}+u_{2}$. And take it into Equation (3.16). Then there comes:

$$
\begin{gathered}
\frac{\mathrm{d}^{2} u_{1}}{\mathrm{~d} \varphi^{2}}+u_{1}=\left(\frac{G M}{L}\right)^{2} \\
\frac{\mathrm{d}^{2} u_{2}}{\mathrm{~d} \varphi^{2}}+u_{2}=\frac{6}{C^{2}}\left(\frac{G M}{L}\right)^{4} e \cos \varphi
\end{gathered}
$$

The solution of Equation (3.17) is:

$$
u_{1}=\left(\frac{G M}{L}\right)^{2}(1+e \cos \theta)
$$

The solution of Equation (3.18) is:

$$
u_{2}=\frac{3}{C^{2}}\left(\frac{G M}{L}\right)^{4} e \varphi \sin \varphi
$$

Hence the solution of Equation (3.16) can be written as:

$$
u=\left(\frac{G M}{L}\right)^{2}\left[1+e \cos \varphi+\frac{3}{C^{2}}\left(\frac{G M}{L}\right)^{2} e \varphi \sin \varphi\right]
$$

By using the auxiliary angle formula as below: 


$$
\begin{aligned}
& a \sin x+b \cos x=\sqrt{a^{2}+b^{2}} \cos (x-\theta) \\
& \theta=\arctan \frac{a}{b} \approx \frac{a}{b} .
\end{aligned}
$$

To ignore part $\left(\frac{G M}{L}\right)^{4}$, the Equation (3.19) can be written as:

$$
u \approx\left(\frac{G M}{L}\right)^{2}\left\{1+e \cos \left[1-3\left(\frac{G M}{c L}\right)^{2}\right] \varphi\right\}
$$

(3.20) is the solution of the planet orbit equation, which is conducted from the modified Newton's theory.

From Equation (3.20), we can see that after running one turning $\varphi=2 \pi$, the planet can't go back to the base point. It will turn a small angle. The reason is that the cycle $T$ of the orbit is no longer $\varphi=2 \pi$. It is:

$$
T=\frac{2 \pi}{1-\frac{3}{C^{2}}\left(\frac{G M}{L}\right)^{2}} .
$$

For the continuous running, at each point, the planet will turn an angle $\varphi_{n}$ to return the base point:

$$
\varphi_{n}=\frac{2 n \pi}{1-\frac{3}{C^{2}}\left(\frac{G M}{L}\right)^{2}} \approx 2 n \pi\left[1+\frac{3}{C^{2}}\left(\frac{G M}{L}\right)^{2}\right]
$$

It means the orbit will have precession. For the orbit with precession, the meaning of base point is not clear. And this precession is not easy to be observed. It can only be observed at perihelion point. From Equation (3.21), we have

$$
\Delta \varphi_{n}=\left(\varphi_{n+1}-\varphi_{n}\right)-2 \pi=6 \pi\left(\frac{G M}{C L}\right)^{2}=6 \pi\left(\frac{G M}{C * r * V}\right)^{2}
$$

The distance from Mercury to the Sun is: $r=4.6 \times 10^{10} \mathrm{~m}$; The Mercury speed at perihelion is: $V=5.8976 \times 10^{5} \mathrm{~m}$; The gravitational constant is: $G=6.67 \times$ $10^{-11} \mathrm{~N} . \mathrm{m}^{2} / \mathrm{kg}^{2}$; The light speed is: $C=2.9979 \times 10^{8} \mathrm{~m} / \mathrm{s}$. Take above date into (3.22), then we have:

$$
\Delta \varphi=0.1^{\prime \prime} .
$$

It means, for every turn, the orbit will have $0.1^{\prime \prime}$ as precession angle. In 100 years, the total precession angle is:

$$
\sum \Delta \varphi \approx 43^{\prime \prime} \text {. }
$$

This result is the same as the observation result, and is the same as the result calculated from General Relativity Theory [5] [6].

\section{To Calculate the Light Deflection Angle}

Assume light, like particles, will be attracted by the sun and will be deflected 
from strait line. According to the modified Newton's theory, the orbit equation of light can be conducted from Equation (3.9), (3.10).

$$
\begin{gathered}
\frac{\mathrm{d} \varphi}{\mathrm{d} t}=\frac{L}{r^{2}} \\
\frac{1}{2}\left(\frac{\mathrm{d} r}{\mathrm{~d} t}\right)^{2}=E+\frac{G M}{r^{2}}\left(1+\frac{V^{2}}{C^{2}}+\frac{V^{4}}{4 C^{4}}\right)-\frac{L^{2}}{2 r^{2}}
\end{gathered}
$$

Take light speed C into Equation (3.10), then there is:

$$
\frac{1}{2}\left(\frac{\mathrm{d} r}{\mathrm{~d} t}\right)^{2}=E+\frac{2.25 G M}{r^{2}}-\frac{L^{2}}{2 r^{2}}
$$

Combine Equation (3.9) and (4.1) and take $\mathrm{d} r=-r^{2} \mathrm{~d}\left(\frac{1}{r}\right)$ into (4.1) then there is:

$$
\frac{L^{2}}{2}\left[\frac{\mathrm{d}\left(\frac{1}{r}\right)}{\mathrm{d} \varphi}\right]^{2}=E-\frac{L^{2}}{2 r^{2}}+\frac{2.25 G M}{r}
$$

To derivative the above formula at both sides, then we have

$$
L^{2} \frac{\mathrm{d}^{2}\left(\frac{1}{r}\right)}{\mathrm{d} \varphi^{2}}+\frac{L^{2}}{r}=2.25 G M
$$

To replace the variable as below:

$$
u=\frac{G M}{r} .
$$

Equation (3.3) can be simplified as:

$$
\frac{\mathrm{d}^{2} u}{\mathrm{~d} \varphi^{2}}+u=2.25\left(\frac{G M}{L}\right)^{2} .
$$

$L=R C . R$ is the radial of the sun; $C$ is the light speed.

To replace the variable again as below:

$$
\begin{aligned}
& \tilde{u}=\frac{1}{r} \text { and } u=\frac{G M}{r}=G M \tilde{u} . \\
& \frac{\mathrm{d}^{2} \tilde{u}}{\mathrm{~d} \varphi^{2}}+\tilde{u}=\frac{2.25 G M}{L^{2}}=\frac{2.25 G M}{(R C)^{2}}
\end{aligned}
$$

To ignore the small quantity $\frac{2.25 G M}{(R C)^{2}}$.

The approximate solution for Equation (3.4) is a strait line.

$$
\tilde{u}_{0}=R^{-1} \cos \varphi
$$

The solution of Equation (4.4) is a line with deflection:

$$
\tilde{u}=\tilde{u}_{0}+\frac{2.25 G M}{(R C)^{2}} \text {. }
$$




$$
\tilde{u}=R^{-1} \cos \varphi+\frac{2.25 G M}{(R C)^{2}}
$$

Strait line (4.5) can be extended to far away.

$$
r \rightarrow \infty ; u \rightarrow 0 \text {. }
$$

The azimuth angle of strait line is $\left( \pm \frac{\pi}{2}\right)$.

At infinite point $(u \rightarrow 0)$, let line (4.6) have azimuth angle as:

$$
\pm\left(\frac{\pi}{2}+\theta\right)
$$

Take it into Equation (3.6)

$$
0=R^{-1} \cos \left[ \pm\left(\frac{\pi}{2}+\theta\right)\right]+\frac{2.25 G M}{C^{2} R^{2}}
$$

Then we can calculate the difference in azimuth angle

$$
\theta \approx \sin \theta=\frac{2.25 G M}{C^{2} R} .
$$

The light deflection angle is

$$
\Delta \varphi=2 \theta \approx \frac{4.5 G M}{C^{2} R}
$$

Take the mass of the sun $M=1.988 \times 10^{30} \mathrm{~kg}$, and radial $R=6.955 \times 10^{8} \mathrm{~m}$ into Equation (4.7), then we have:

$$
\Delta \varphi=1.97 " .
$$

This result is much close to the observation data of $1.89 "$, with deviation of +0.08 " from the observation data. The same result calculated from general relative theory is $1.75 "$ ", with deviation of -0.1 " from observation data. [5] [6]. The summary of the calculation result is as below:

Light deflection angle from observation data: $1.89 "$.

Light deflection angle from Relative theory: 1.75 "; with deviation of -0.11 ".

Light deflection angle from this paper: 1.97 "; with deviation of +0.08 ".

\section{The Gravitational Force for the Two Objectives with Zero Related Speed}

According to Equation (2.2)

$$
F=\frac{G M m}{r^{2}}\left(1+\frac{V^{2}}{C^{2}}+\frac{V^{4}}{4 C^{4}}\right)
$$

When the related speed of the two objectives is $V=0$, this equation will be the same as Newton's gravitational force theory.

$$
F=\frac{G M m}{r^{2}}\left(1+\frac{0^{2}}{C^{2}}+\frac{0^{4}}{4 C^{4}}\right)=\frac{G M m}{r^{2}} .
$$

\section{Conclusions}

According to the above 3 examples, we tested the modified Newton's theory for 
3 different relative speeds, i.e. for objectives with relative zero speed, with light speed and with low speed (Mercury running orbit).

When introducing speed $V$ into Newton's gravitational theory, we can conduct the same orbit equation for mercury orbit and light deflection. And the modified Newton's theory will become the same as Newton's gravitational theory when the objectives speed is zero. These results are also the same as the results calculated from the general relativity theory.

It is concluded that the gravity force is coming from the total energy of objects, instead of from the mass of objects. And Modified Newton's Gravity theory and General Relative theory have the same accuracy to calculate planets orbit and light deflection.

\section{Conflicts of Interest}

The author declares no conflicts of interest.

\section{References}

[1] Zhou, Y.B. (2011) Theoretical Mechanics. Higher Education Press, Beijing, 50-53.

[2] Chen, S.M. (2018) The Dynamic Theory. Higher Education press, Beijing, 49-50.

[3] Bootello, J. (2012) Angular Precession of Elliptic Orbits. Mercury. International Journal of Astronomy and Astrophysics, 2, 249-255. https://doi.org/10.4236/ijaa.2012.24032

[4] De Matos, C.J. and Tajmar, M. (2000) Advance of Mercury Perihelion Explained by Cogravity. Oalib Physics. https://arxiv.org/ftp/gr-qc/papers/0005/0005040.pdf

[5] Zhao, Z. (2018) The Foundation of General Gravitational Theory. Tsinghua University Press, Beijing, $94 \mathrm{p}$.

[6] Chen, B. (2020) General Gravitational Theory. Beijing University Press, Beijing, 313 p. 